\title{
Increased Expression of HOXB2 and HOXB13 Proteins is Associated with HPV Infection and Cervical Cancer Progression
}

\author{
AL Gonzalez-Herrera ${ }^{1}$, M Salgado-Bernabe ${ }^{1}$, CK Velazquez-Velazquez ${ }^{1}, M$ \\ Salcedo-Vargas ${ }^{3}$, A Andrade-Manzano ${ }^{2}$, F Avila-Moreno ${ }^{4}$, P Pina-Sanchez ${ }^{1 *}$
}

\begin{abstract}
Background: Cervical cancer ( $\mathrm{CeCa})$ is the second most common cancer in women in developing countries, and human papilloma virus (HPV) is the primary etiological factor. Aberrant expression of HOX transcription factors has been observed in several types of cancer. To date, however, no reports exist on the expression of HOXB2 and HOXB13 proteins during neoplastic progression in $\mathrm{CeCa}$ and its correlation with HPV infection. Materials and Methods: Expression of HOXB2 and HOXB13 proteins was assessed in tissue microarrays from normal cervical epithelium, cervical intraepithelial neoplasias grade 1-3, and CeCa. HPV was detected by PCR and sequencing. Expression of HOX-positive cells was determined in each diagnostic group. Results: Percentage of HOXB2- and HOXB13-positive cells gradually increased from means of $10.9 \%$ and $16.7 \%$, respectively, in samples from healthy women, to $\mathbf{7 5 . 2 \%}$ and $\mathbf{8 8 . 6 \%}$ in those from CeCa patients. Frequency of HPV infection also increased from $13 \%$ in healthy tissue samples to $92.3 \%$ in CeCa. Both HOXB2 and HOXB13 proteins were preferentially expressed in HPV+ samples. Conclusions: The present study represents the first report on the expression of both $\mathrm{HOXB} 2$ and $\mathrm{HOXB13}$ proteins through cervix tumorigenesis, providing evidence that increased expression of such proteins is a common event during progression to $\mathrm{CeCa}$.
\end{abstract}

Keywords: Cervical cancer - HOXB2 and HOXB13 proteins - HPV infection - progression

Asian Pac J Cancer Prev, 16 (4), 1349-1353

\section{Introduction}

HOX genes are transcription factors that possess a conserved sequence of 183 nucleotides coding for a homeodomain of 61 amino acids. Such a sequence is important for HOX protein interaction with DNA (Ghering, 1987; Gehring et al., 1990). HOX genes have been identified as key players in embryonic development, participating in cell differentiation and body positioning along the anteroposterior axis (Krumlauf, 1994). Humans have 39 HOX genes grouped in four clusters (HOXA, HOXB, HOXC and HOXD, located on chromosomes 7, 17,2 and 12, respectively) with nine to eleven genes in each cluster (Favier and Dolle, 1997). Three genes of the HOXA cluster, HOXA10, HOXA11 and HOXA13, have been shown to be involved in the development of female genitourinary structures, and this occurs via estradiol (Daftary and Taylor, 2006). Interestingly, teratogen effects have been described which are caused by diethylstilbestrol during development of the female reproductive tract, due to the suppression of the expression of such abdominal HoxA genes (Ma et al., 1998).

A wide variety of alterations in the expression of HOX genes have been reported in different solid neoplasms and leukemias, some of which have been recognized as important parts of the oncogenic process (Shah and Sukumar, 2010. In this sense, HOX alterations have been proposed as biomarkers for the diagnosis and prognosis -and as a potential target for therapy- in several types of cancer (Shah and Sukumar, 2010). It is noteworthy, however, that their particular role in cervical cancer $(\mathrm{CeCa})$, if any, remains to be elucidated.

According to WHO reports, $\mathrm{CeCa}$ represents the third most common cause of death in women. In 2012, 527,624 new cases and 265,653 deaths were reported. Eighty five percent of cases occur in developing countries, representing a serious public health problem, in spite of the presence of screening programs (Ferlay et al., 2014; Ali et al., 2012). The main etiological factor for $\mathrm{CeCa}$ is a persistent infection with high-risk of the Human Papilloma Virus (HPV) (zur Hausen 2009). Evidence exists, however, indicating that HPV infection is not enough for cancer to develop, and other cofactors, such as hormonal contraceptive, smoking, parity and number of sexual partners, seem to be required (Luhn, et al., 2013). Moreover, a large number of molecular alterations have 
been described, including abnormal gene copy numbers (Hidalgo et al., 2005; Polocht et al., 2010; Kuglif et al. 2014;) and expression profiles (Perez-Plasencia et al., 2007).

Several studies, at the mRNA level, have shown altered expression of different HOX genes in $\mathrm{CeCa}$ and $\mathrm{CeCa}$ derived cell lines, as compared to normal epithelium. Indeed, Shim and colleagues observed overexpression of HOXA1 (Shim et al., 1998), whereas Lopez et al found overexpression of HOXB2, HOXB4 and HOXB13 (Lopez et al., 2006). Moreover, HOXC5 and HOXC8 overexpression has been described in different $\mathrm{CeCa}$ cell lines (Alami et al., 1999). However, to date, only three reports have been published on the expression of $\mathrm{HOX}$ proteins in $\mathrm{CeCa}$, namely HOXB4 (Barba de la Rosa et al., 2012), HOXD13 (Cantile et al., 2009), and HOXC10 (Zhai et al., 2007). Among them, only the expression of HOXB4 protein was assessed in normal epithelium, precursor lesions, and CeCa (Barba de la Rosa et al., 2012). A functional study demonstrated that human foreskin keratinocytes transformed with HPV16-E6/ E7 showed alterations in EZH2 (PRC2) and KDM6A (PRC1) that were associated to lack of repression of HOX genes (Hayland et al., 2011). Taken together, the above mentioned reports indicate that overexpression of HOX genes seems to be involved in the tumor progression of $\mathrm{CeCa}$.

To date, however, the expression profiles of $\mathrm{HOX}$ proteins in the context of HPV-associated $\mathrm{CeCa}$ progression remain to be assessed. Thus, the main objective of the present study was to determine the expression profiles, at the protein level, of HOXB2 and HOXB13 in 209 samples of CeCa, CIN1-3, and normal cervical epithelium. Our results show overexpression of both HOXB2 and HOXB13 in CIN2-3 and CeCa; however, only HOXB13 is associated to oncogenic HPV infection.

\section{Materials and Methods}

\section{Tissue Microarray (TMA) construction}

Formalin-Fixed and Paraffin-Embedded (FFPE) tissue samples were collected from the pathology archive at "Luis Castelazo" Gynecology Hospital, IMSS, Mexico City. Samples from patients with squamous cervical cancer or patients with preneoplastic lesions, i.e., cervical intraepithelial neoplasia grades 1, 2, and 3, as well as samples from normal histological epithelium (without neoplastic alterations), were selected. Microtome slices $(4 \mu \mathrm{m})$ were obtained using a Leica RM2125 Microtome (Wetzlar, Germany) and were stained with hematoxylineosin; slices were reviewed by an experienced pathologist and representative areas were identified for the construction of tissue micro-array (TMA). The selected tissue sections were punched with a $1 \mathrm{~mm}$ needle, and transferred to an acceptor block using Tissue Microarrayer ATA 100 Chemicon (Temecula CA). Tissue sections were obtained from TMA and transferred to slide glass (VWR Superfrost Plus) for immunohistochemistry assays.
The TMA slides were processed in a Ventana Benchmark system (Roche). The primary antibodies used were HOXB2, (Santa Cruz Biotechnology, Santa Cruz CA; 1:200 dilution) and HOXB13 (Santa Cruz Biotechnology, Santa Cruz CA; 1:50 dilution). As a negative control, the primary antibody was replaced with a normal rabbit IgG. The UltraView Universal DAB detection kit was used (Ventana Medical System Inc., Tucson AZ); the slides were dehydrated using ascending-alcohol solutions; cleared in a xylol solution, and mounted in synthetic resin. TMAs were observed in a DM750 Leica microscope (Wetzlar, Germany), and digital images were obtained using the LEZ software; two independent observers evaluated the reaction. The immunostaining intensity was scored in terms of the percentage of positive cells.Data were analyzed using the PASW statistics package 18 (IBM Corp., Armonk, NY, USA).

\section{HPV Detection}

Microtome sections $(60 \mu \mathrm{m})$ from paraffin blocks were deparaffinized with xylene and absolute ethanol; genomic DNA was extracted using Wizard genomic DNA Purification kit (Promega, CA, USA). DNA was quantified in NanoDrop Spectrophotometer (Delaware, USA). HPV detection was performed as described previously (Piña et al., 2006).

\section{Statistical analysis}

Data were collected using the PASW Statistics software, version 18 (IBM Corp., Armonk, NY, USA). The Kolmogorov-Smirnoff test was used to determine the distribution of quantitative variables. We performed a bivariate analysis of the percentage of cells that were positive to HOXB2 and HOXB13 proteins, and HPV positive samples in each diagnosis group. U Mann-Whitney test was used for statistical differences. Multivariate analysis and logistic regression were performed to determine protein expression and HPV infection adjusted for diagnosis group, Odds ratio (OR) and a $95 \%$ confidence interval were also calculated.

\section{Results}

HOXB2 and $H O X B 13$ overexpression in cervical carcinoma progression

Two hundred and nine samples obtained from TMA were analyzed, including 54 from normal cervical tissues (control), 65 from CIN grade 1, 25 from CIN grades 2-3, and 65 from CeCa. As shown in Figure 1A, a consistent, progressive increase in protein expression was observed, both for HOXB2 (from 10.9\% positive cells in control epithelium to $75 \%$ positive cells in $\mathrm{CeCa}$ ) and $\mathrm{HOXB} 13$ ( $16.7 \%$ positive cells in control samples, and $88.6 \%$ positive cells in $\mathrm{CeCa}$ ). It is noteworthy that protein expression in control samples was mainly localized in the cytoplasm of basal and parabasal cells (Figure 2A, 2D); in contrast, cervical precursor lesions showed expression of HOXB2 and HOXB13 mainly in nuclei, with intense detection in koilocytes (Figure 2B, 2E). Similarly, CeCa samples showed intense stain in the nuclei, particularly for HOXB13 protein (Figures 1B, 2C, 2E, and 2F). For both, 
HOXB2 and HOXB13 significant statistical differences $(p<0.05)$ were observed between most groups, except for HOXB2 when comparing CIN 2-3 and $\mathrm{CeCa}(\mathrm{p}=0.07)$ (Figure 1A).

HPV16 is the most frequent infection associated to $\mathrm{CeCa}$ progression

By using PCR and sequencing, the presence of HPV was found in $13 \%$ of control samples, in $44 \%$ of CIN1 samples, in $88 \%$ of CIN 2-3, samples and in $92 \%$ of CeCa samples (Table 1). The three most frequent HPV types in CeCa were HPV16, 18 and 58. In fact, HPV16 was observed in all diagnostic groups, being most abundant in CIN 2-3 and $\mathrm{CeCa}$ ( $44 \%$ and $40 \%$ of samples, respectively); HPV18, on the other hand, was only found in $\mathrm{CeCa}$ at a low frequency (6.2\% of samples). HPV58 was not detected in control samples, and was identified in $4 \%$ of CIN2-3 samples and in $6.2 \%$ of CeCa samples (Table 1).
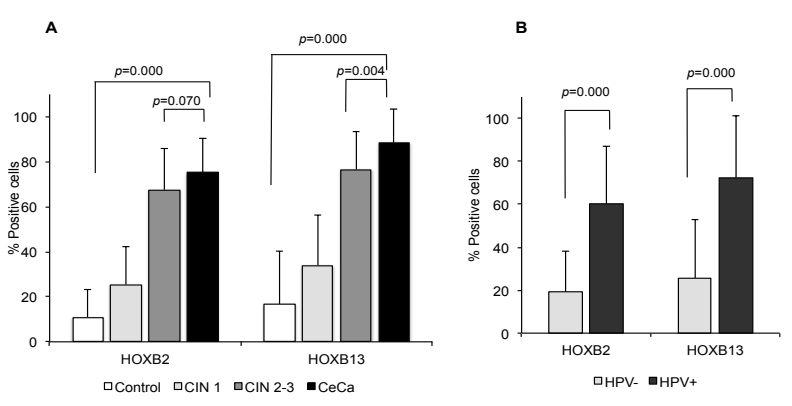

Figure 1. Expression of Proteins HOXB2 and HOXB13 Associated to Cervical Lesions and HPV Infection. Expression of HOXB2 and HOXB13 proteins in epithelium without neoplastic alterations (control) CIN 1, CIN 2-3 and cervical cancer $(\mathrm{CeCa})$. The average was calculated based on percentage of positive cells in each sample (A). Percentage of expression of HOXB2 and HOXB13 proteins according to presence of HPV infection (B). U Mann-Whitney test was used for statistical differences

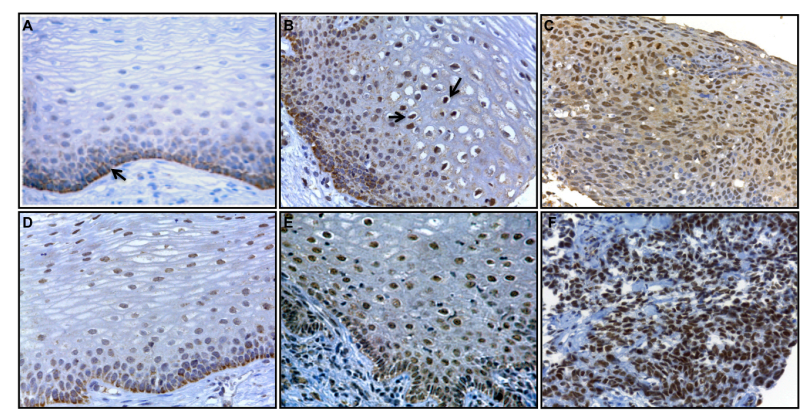

Figure 2. Expression of HOXB2 and HOXB13 in Cervical Epithelium. A) Expression of HOXB2 protein, the reaction is localized in cytoplasm of basal and parabasal cells as shown in the arrow. B) CIN1 with HOXB2 expression in nuclei of basal, parabasal and intermediate cells, the arrows shown koilocytes with high nuclear expression. C) Presence of $\mathrm{HOXB} 2$ protein in nuclei and cytoplasmic of $\mathrm{CaCu}$ sample. D) Normal epithelium showing HOX13 in cytoplasm of basal cell E) CIN3, almost all cells have expression of HOXB13 protein in nuclei F) Invasive cervical cancer showing the HOXB13 protein expression in epithelial cell nuclei
Table 1. Detection of HPV in Cervical Intraepithelial Neoplastic, Epithelium without Neoplastic Lesion and Cervical Cancer

\begin{tabular}{lcccccccr}
\hline & \multicolumn{2}{c}{ Control } & \multicolumn{2}{c}{ CIN 1 } & \multicolumn{2}{c}{ CIN 2-3 } & \multicolumn{2}{c}{ CeCa } \\
& $\mathrm{n}=54$ & $\%$ & $\mathrm{n}=65$ & $\%$ & $\mathrm{n}=25$ & $\%$ & $\mathrm{n}=65$ & $\%$ \\
\hline HPV- & 47 & 87.0 & 36 & 55.4 & 3 & 12.0 & 5 & 7.7 \\
HPV+ & 7 & 13.0 & 29 & 44.6 & 22 & 88.0 & 60 & 92.3 \\
HPV16 & 3 & 5.6 & 10 & 15.4 & 11 & 44.0 & 26 & 40.0 \\
HPV18 & - & - & - & - & - & - & 4 & 6.2 \\
HPV58 & - & - & 1 & 1.5 & 1 & 4.0 & 4 & 6.2 \\
Others & 4 & 7.4 & 18 & 27.7 & 10 & 40.0 & 26 & 40.0 \\
\hline *CCa: Cervical cancer. CIN: cervical intraepithelial neoplasia
\end{tabular}

Table 2. Multivariate Analysis Showing HOXB2 and HOXB13 Protein Expression Associated to HPV Infection

\begin{tabular}{llrrrc}
\hline $\begin{array}{l}\text { Diagnosis } \\
\text { group }\end{array}$ & Protein & OR & \multicolumn{2}{c}{ CI 95\% } & p \\
\hline Control & & & Lowest & Highest & \\
CIN 1 & HOXB2 & 1 & & & \\
& HOXB13 & 1.4 & 2.7 & 27.1 & $0.00^{*}$ \\
CIN2-3 & HOXB2 & 173.9 & 17.3 & 47.7 & 0.54 \\
& HOXB13 & 52.3 & 8.6 & 315.6 & $0.00^{*}$ \\
CeCa & HOXB2 & 443.2 & 44.7 & 4393.5 & $0.00^{*}$ \\
& HOXB13 & 87.5 & 17.9 & 428.4 & $0.00^{*}$ \\
HPV- & & 1 & & & \\
HPV+ & HOXB2 & 2.3 & 0.9 & 5.6 & 0.07 \\
& HOXB13 & 3.46 & 1.3 & 9.4 & $0.016^{*}$ \\
\hline
\end{tabular}

*The expression was dichotomized according to median percentage of protein expression, for HOXB $2<30 v s \geq 30$, and HOXB13 $\leq 50 v s>50$. *Statistical significance

HOXB2 and HOXB13 overexpression is associated to oncogenic HPV infection in cervical precursor and $\mathrm{CaCu}$ lesions

To determine the potential relationship between HOXB2 and HOXB13 overexpression with HPV infection, we determined the percentage of cells expressing anyone of the two HOX proteins together with the presence or absence of HPV, regardless of the diagnosis group. As shown in Figure 1B, both HOXB2 and HOXB13 were preferentially expressed in HPV-positive samples. Indeed, in both cases the percentage of cells expressing anyone of the HOX proteins was 3-fold higher in HPV-positive samples, as compared to HPV-negative samples. In addition, to determine whether the increased expression of HOXB2 and HOXB13 was associated to HPV infection and diagnosis, a multivariate analysis was performed. Table 2 shows that HOXB2 protein was significantly expressed in all patient groups -CIN1, CIN 2-3, and $\mathrm{CeCa}(\mathrm{p}<0.05)$ - whereas HOXB13 protein was significantly expressed only in CIN2-3 and CeCa. $(\mathrm{p}<0.05)$. Interestingly, only HOXB13 protein expression was associated to HPV positive samples $(\mathrm{p}=0.016)$.

\section{Discussion}

Previous reports from our group demonstrated, at the mRNA level, the expression of HOXB2 and HOXB13 in $\mathrm{CeCa}$, in contrast to normal cervical epithelium; thus, suggesting its involvement in the development of 
histopathological progression of $\mathrm{CeCa}$ (Lopez et al., 2006). To date, however, HOXB2 and HOXB13 have not been studied, at the protein level, in preneoplastic lesions and cervical cancer.

In this study, we found that a low proportion of cells from normal cervical epithelium expressed HOXB2 or HOXB13 proteins. Such proteins were mainly expressed in the cytoplasm of cells of the basal layer. To date, however, the function of HOXB2 and HOXB13 proteins in normal cervix epithelium is unknown. Other groups have shown that HOXB13 participates in ventral prostate (Economides et al., 2003) and epidermal differentiation (Mack et al., 2005). On the other hand, HOXB2 seems to be involved in early neurogenesis, specifically in the development of motor neurons (Davenne et al., 1999). The role of these HOX proteins in normal cervical epithelium remains to be determined.

In the present study, we detected HOXB2 and HOXB13 proteins in the nuclei of cells from cervical intraepithelial lesions and $\mathrm{CeCa}$ samples. Such a nuclear location could be related to interactions with cofactors such MEIS, PBX and PREP (Mann 2009). It is noteworthy that for the first time, a gradual increase in HOXB2 protein expression was documented, from normal cervical epithelium and CIN 1 to CIN 2-3 and CeCa. This is in keeping with previous studies showing that increased HOXB2 mRNA expression in epithelial tumors, such as pancreatic (Segara et al., 2005) and lung cancer (Inamura et al., 2007; Inamura et al., 2008) are associated with poor prognosis, promoting invasion and regulation of metastasis-related genes.

HOXB13 has been studied in several cancer types. In ER+ Breast cancer, some studies have shown that the expression ratio of HOXB13:IL17R is a predictor biomarker for Tamoxifen therapy, and is associated to poor prognosis (Ma et al., 2006; Zhao 2014). One of the mechanisms involved in Tamoxifen chemoresistance is associated to high levels of HOXB13, which acts by down-regulating ESR1 through NCOR corepressor; and by up-regulating IL-6 through PBX1 and MEIS1 cofactors (Shah, 2013). In ovarian cancer, HOXB13 promotes proliferation and participates in the development of Tamoxifen resistance in ER+ patients (Miao et al. 2007). In prostate cancer, HOXB13 overexpression has been documented (Kim et al., 2010). One of the mechanisms proposed for HOXB13-induced metastasis is by upregulation of Zn transporter (ZnT4), reducing intracellular $\mathrm{Zn}$ and stimulating expression of IKK $\alpha$, which activates genes like MMP-9 and VEGF, through p65 and p50 (Kim et al, 2013). In CeCa, MMP-9 participation has been documented in invasion and metastasis (Weiwei, 2009). Whether such an induction of MMP-9 is due to HOXB13 remains to be determined.

HOXB13 protein expression, however, has not been studied in $\mathrm{CeCa}$. In the present study, we observed a significant increase in the frequency of cells expressing HOXB13 protein throughout cervical carcinoma progression. Additionally, overexpression of HOXB13 was significantly associated to HPV high-risk infection. These findings are in keeping with a recent report by Hayland et al, in which they observed increased expression and phosphorylation of EZH2Ser21 (PCR2) in keratinocytes, which was induced by the E6/E7 oncogenes from HPV16 (Hayland et al., 2011). Unexpectedly, an increase of $\mathrm{H} 3 \mathrm{~K} 27 \mathrm{me} 3$ was not detected, and derepression of HOXC5, HOXC8, HOXA7, HOXA9 and HOXA11 was observed (Hayland et al., 2011). In such a study, however, HOXB2 and HOXB13 were not evaluated. These findings suggest a relationship between HPV oncoproteins and deregulated expression of HOX genes. However the actual mechanisms remain to be elucidated.

These data suggest that deregulated expression of HOXB13 is a common mechanism in hormone-related cancer, such as breast and prostrate cancer. Although $\mathrm{CeCa}$ is not directly related to hormonal causes, the persistent hormonal consumption is recognized as a cofactor for CeCa development (Luhn et al., 2013); furthermore, in the K14E7 murine model of $\mathrm{CeCa}$, ER positivity has been identified as an indispensable factor for carcinogenesis (Chung et al., 2008).

In conclusion, the present study represents the first report focus on the expression of both, HOXB2 and $\mathrm{HOXB} 13$ proteins throughout $\mathrm{CeCa}$ progression, and shows that increased expression of HOXB2 and HOXB13 proteins is a common event during the histopathological progression of $\mathrm{CeCa}$. Whether overexpression of these HOX proteins is a necessary step for neoplastic transformation remains to be determined. Our study also demonstrated a positive association between altered HOXB2 and HOXB13 protein expression and oncogenic HPV infection. Functional assays are necessary for understanding the involvement of HOXB13 and HOXB2 in the development of $\mathrm{CeCa}$.

\section{Acknowledgements}

This work was partially supported by the National Council of Science and Technology (CONACYT, Mexico; grant 87474) and the Mexican Institute for Social Security (IMSS; grant FIS/IMSS/PROT/529), as well as grants from the National Autonomous University of Mexico (UNAM; grants PAPIIT IB202512 and PAPIIT RR282512). The authors thank Dr. Rafael Arias for his statistical support, and Dr.Hector Mayani for his scientifically comments and criticisms in the review of this manuscript.

\section{References}

Alami Y, Castronovo V, Belotti D, et al (1999). HOXC5 and HOXC8 expression are selectively turned on in human cervical cancer cells compared to normal keratinocytes. Biochem Biophys Res Commun, 257, 738-45.

Ali F, Kuelker R and Wassie B (2012). Understanding cervical cancer in the context of developing countries. Ann Trop Med $P H, \mathbf{5}, 3$.

Barba-de la Rosa AP, Briones-Cerecero E, Lugo-Melchor O, et al. (2012). Hox B4 as potential marker of non-differentiated cells in human cervical cancer cells. J Cancer Res Clin Oncol, 138, 293-300.

Cantile M, Franco R, Tschan A, et al (2009). HOX D13 expression across 79 tumor tissue types. Int J Cancer, $\mathbf{1 2 5}$, 1532-41.

Chung SH, Wiedmeyer K, Shai A, et al (2008). Requirement for estrogen receptor $\alpha$ in a mouse model for human 
papillomavirus-associated cervical cancer. Cancer Res, 68, 9928-34.

Daftary GS and Taylor HS (2006). Endocrine regulation of HOX genes. Endocr Rev, 27, 331-55.

Davenne M, Maconochie MK, Neun R, et al (1999). Hoxa2 and Hoxb2 control dorsoventral patterns of neuronal development in the rostral hindbrain. Neuron, 4, 22.

Economides KD, and Capecchi MR (2003). Hoxb13 is required for normal differentiation and secretory function of the ventral prostate. Development, 130, 2061-9.

Favier B and Dolle P (1997). Developmental functions of mammalian Hox genes. Mol Hum Reprod, 3, 115-131.

Ferlay J, Soerjomataram I, Ervik M, et al (2014). GLOBOCAN 2012 v1. 0, Cancer incidence and mortality worldwide: IARC CancerBase No. 11 [Internet]. Lyon, France: International agency for research on cancer. c2013 [cited 2013 Oct 17]. globocan. iarc. fr.

Gehring WJ (1987). Homeoboxes in the study of development. Science, 236, 1245-52

Gehring WJ, Muller M, Affolter M et al (1990). The structure of the homeodomain and its functional implications. Trends Genet, 6, 323-9.

Hidalgo A, Baudis M, Petersen I, et al (2005). Microarray comparative genomic hybridization detection of chromosomal imbalances in uterine cervix carcinoma. BMC Cancer, 5, 77.

Hyland PL, McDade S, McCloskey R, et al (2011). Evidence for alteration of EZH2, BMI1, and KDM6A and epigenetic reprogramming in human papillomavirus type $16 \mathrm{E} 6 / \mathrm{E} 7-$ expressing keratinocytes. J Virology, 85, 10999-11006.

Inamura $\mathrm{K}$, Togashi $\mathrm{Y}$, Ninomiya $\mathrm{H}$, et al (2008). HOXB2, an adverse prognostic indicator for stage I lung adenocarcinomas, promotes invasion by transcriptional regulation of metastasis-related genes in HOP-62 non-small cell lung cancer cells. Anticancer Res, 28, 2121-7.

Inamura K, Togashi Y, Okui M, et al (2007). HOXB2 as a novel prognostic indicator for stage I lung adenocarcinomas. $J$ Thorac Oncol, 2, 802-7.

Kim SD, Park RY, Kim Y, et al (2010). HOXB13 is co-localized with androgen receptor to suppress androgen-stimulated prostate-specific antigen expression. Anat Cell Biol, 43, 284-93.

Kim YR, Kim IJ, Kang TW, et al (2013). HOXB13 downregulates intracellular zinc and increases NF- $\varkappa \mathrm{B}$ signaling to promote prostate cancer metastasis. Oncogene, 33, 4458-67.

Krumlauf R (1994). Hox genes in vertebrate development. Cell, 29, 191-201.

Kuglik P, Smetana J, Vallova V, et al (2014). Genome-wide screening of DNA copy number alterations in cervical carcinoma patients with CGH+ SNP microarrays and HPVFISH. Int J Clin Exp Pathol, 7, 5071.

Lopez R, Garrido E, Pina P et al (2006). HOXB homeobox gene expression in cervical carcinoma. Int J Gynecol Cancer, 16, 329-35.

Luhn P, Walker J, Schiffman M, et al (2013). The role of cofactors in the progression from human papillomavirus infection to cervical cancer. Gynecol Oncol, 128, 265-270.

Ma L, Benson GV, Lim H et al (1998). Abdominal B (AbdB) hoxagenes: regulation in adult uterus by estrogen and progesterone and repression in mullerian duct by the synthetic estrogen diethylstilbestrol (DES). Dev Biol, 197, 141-54.

Ma XJ, Hilsenbeck SG, Wang W et al (2006). The HOXB13: IL17BR expression index is a prognostic factor in early-stage breast cancer. J Clin Oncol, 24, 4611-9.

Mack JA, Li L, Sato N et al (2005). Hoxb13 up-regulates transglutaminase activity and drives terminal differentiation in an epidermal organotypic model. J Biol Chem, 280, 29904-11.

Mann R S, Lelli KM, Joshi R (2009). Hox specificity: unique roles for cofactors and collaborators. Curr Top Dev Biol, 88, 63-101.

Miao J, Wang Z, Provencher H, et al (2007). HOXB13 promotes ovarian cancer progression. Proc Nat Acad Sci USA, 104, 17093-8.

Pérez-Plasencia C, Vazquez-Ortiz G, Lopez-Romero R, et al (2007). Genome wide expression analysis in HPV16 cervical cancer: identification of altered metabolic pathways. Infect Agent Cancer, 2, 16.

Piña Sanchez P, Hernandez-Hernandez DM, Lopez-Romero R, et al (2006). Human papillomavirus-specific viral types are common in Mexican women affected by cervical lesions. Int J Gynecol Cancer, 16, 1041-7.

Policht FA, Song M, Sitailo S. et al (2010). Analysis of genetic copy number changes in cervical disease progression. $B M C$ cancer, 10, 432.

Segara D, Biankin AV, Kench JG, et al (2005). Expression of HOXB2, a retinoic acid signaling target in pancreatic cancer and pancreatic intraepithelial neoplasia. Clin Cancer Res, 11, 3587-96.

Shah N, Sukumar S. (2010). The Hox genes and their roles in oncogenesis. Nature Reviews Cancer, 10, 361-71.

Shah N, Jin K, Cruz LA, et al (2013). HOXB13 mediates tamoxifen resistance and invasiveness in human breast cancer by suppressing ER $\alpha$ and inducing IL-6 expression. Cancer Res, 73, 5449-58.

Shim C, Zhang W, Rhee CH, et al (1998). Profiling of differentially expressed genes in human primary cervical cancer by complementary DNA expression array. Clin Cancer Res, 4, 3045-50.

Walboomers JM, Jacobs MV, Manos M. et al (1999). Human papillomavirus is a necessary cause of invasive cervical cancer worldwide. J Pathol, 189, 12-9.

Weiwei Y, Jinhui L, Xiaoliang X, et al (2009). Expression of MMP9 and CD147 in invasive squamous cell carcinoma of the uterine cervix and their implication. Pathol Res Practice, 205, 709-15.

Zhai Y, Kuick, R, Nan B, et al (2007). Gene expression analysis of preinvasive and invasive cervical squamous cell carcinomas identifies HOXC10 as a key mediator of invasion. Cancer Res, 67, 10163-72.

Zhao L, Zhu S, Gao Y, et al (2014). Two-gene expression ratio as predictor for breast cancer treated with tamoxifen: evidence from meta-analysis. Tumor Biol, 35, 3113-7.

zur Hausen H (2009b). Papillomaviruses in the causation of human cancers - a brief historical account. Virology, 384, 260-5. 